\title{
Photoviscoelastic Stress and Strain Analysis around a Surface Groove under Rolling Contact Load*
}

\author{
Satoru YONEYAMA**, Daisuke AYAME**, \\ Jin'ichiro GOTOH*** and Masahisa TAKASHI**
}

\begin{abstract}
The authors discuss the first step in the approach to the fundamental mechanism of small-scale local fracture near the contact surface between a rigid traveling roller and a viscoelastic plate which has a deep groove on the upper edge of the plate, which simulates pitting or surface cracking around the contact surface. The photoviscoelastic method is applied to investigate the time-dependent stress and strain state under the condition of nonproportional loading, taking a typical case of the interaction of stress fields due to a traveling contact load and a surface groove into account. Complicated variations of the time-dependent photoviscoelastic fringe pattern are observed near the tip of the surface groove. The time variations of not only the principal stress difference and principal strain difference, but also principal directions of stress, strain and birefringence are evaluated using a convenient computer-aided photoviscoelastic technique under the temperature at which the material shows marked viscoelastic behavior. Time variations of stress intensity factors, $K_{I}$ and $K_{I I}$, are also evaluated using the least ${ }^{-}$squares method following the technique proposed by Sanford and Dally.
\end{abstract}

Key Words : Rolling Contact, Stress Intensity Factor, Viscoelasticity, Photoviscoelasticity, Experimental Stress Analysis

\section{Introduction}

Small-scale local fracture such as pitting and wearing are often seen on the edge surface of a viscoelastic plate or disk under rolling contact load. Microscopic surface damage such as pitting can grow easily into macroscopic cracks under repetition of such circumstances. It should therefore be pointed out that the dominant factor in the process from micropitting to macroscopic cracks will be affected not only by the stress-strain state but their rates of variation under rolling contact load. This type of time-dependent mixed-mode fracture problem has rarely been discussed or investigated up to now from the viewpoint of fracture mechanics for viscoelastic materials. However, in order to establish an appropriate crite-

* Received 4th June, 1996.

** Department of Mechanical Engineering, Aoyama Gakuin University, 6-16-1 Chitosedai, Setagaya-ku, Tokyo 157, Japan

*** Department of Mechanical Engineering and Materials Science, Yokohama National University, 156 Tokiwadai, Hodogaya-ku, Yokohama, Kanagawa 240, Japan rion of crack extension in such situations, an approach which can clarify not only time-dependent stress-strain distribution but basic mechanisms will be indispensable.

As is well known, one approach to viscoelastic stress and strain analysis is based on the direct calculation of the convolution integral over a wide range of time in the framework of the mathematical theory of viscoelasticity. Another one, on the other hand, makes use of the well-known correspondence. principle $^{(1)}$, whereby viscoelastic solutions can be deduced by inverse transformation from the corresponding pseudoelastic solution on a Laplace-transformed surface. It is, however, pointed out that the former is successful in limited cases only of simple geometry or under proportional boundary conditions. In this context, the authors have already developed a computer-aided photoviscoelastic technique for the establishment of an easy, simple method ${ }^{(2)}$ applicable to nonproportional viscoelastic problems, based on the correspondence principle. Also, some examples of the viscoelastic rolling contact problem ${ }^{(3)}$ involving discussions on the possibility of the application of the Hertz theory to viscoelastic contact problem $^{(4)}$ have 
been investigated previously.

In this paper, the authors discuss the evaluation of time-dependent stresses around a deep groove near the contact surface, taking the interaction of stress fields arising from both a rolling contact load and a surface groove into account. Complicated variation of the time-dependent photoviscoelastic fringe pattern near the tip of the surface groove is periodically observed using a CCD camera. The time variations not only of principal stress/strain difference, but also of the principal axis of stress/strain and birefringence are evaluated using the convenient computer-aided photoviscoelastic method, under the temperature at which the material shows marked viscoelastic behavior.

Time variations of stress intensity factors, $K_{I}$ and $K_{I I}$, around the groove tip are also evaluated using the least squares method as the first step in the approach to the fundamental mechanism of small-scale cracking near the contact surface of a viscoelastic plate or disk. The experimental results obtained under a weak effect of friction show fairly good qualitative agreement with those of the current FEM analysis. On the bases of the results of this study, it is emphasized, from the industrial viewpoint, that extraordinary types of wear and/or fracture of polymeric materials under rolling contact load, such as in the cases of the pinch roller of a tape recorder and a rubber tire of an automobile, can be analyzed successfully by the method proposed.

\section{Photoviscoelastic Analysis}

\section{1 Constitutive equations of photoviscoelas- ticity}

According to the generalized photoviscoelastic equations $^{(5)}$ shown below, not only the time-dependent principal stress difference $\sigma_{1}(t)-\sigma_{2}(t)$ and the principal strain difference $\varepsilon_{1}(t)-\varepsilon_{2}(t)$ but also the angle $\beta(t)$ of the principal stress direction and the angle $\gamma(t)$ of the principal strain direction can be calculated, if the time variation of both the fringe order $n(t)$ and the principal birefringence angle $\alpha(t)$ at any point are obtained from photoviscoelastic image data.

$$
\begin{aligned}
& {\left[\sigma_{1}(t)-\sigma_{2}(t)\right] \cos 2 \beta(t)=C_{\sigma r}^{-1}(0) \cdot n(0) \cos 2 \alpha(0)} \\
& \quad+\int_{0}^{t} C_{\sigma r}^{-1}(t-\tau) \frac{d n(\tau) \cos 2 \alpha(\tau)}{d \tau} d \tau \\
& {\left[\sigma_{1}(t)-\sigma_{2}(t)\right] \sin 2 \beta(t)=C_{\sigma r}^{-1}(0) \cdot n(0) \sin 2 \alpha(0)} \\
& \quad+\int_{0}^{t} C_{\sigma r}^{-1}(t-\tau) \frac{d n(\tau) \sin 2 \alpha(\tau)}{d \tau} d \tau
\end{aligned}
$$

$$
\begin{aligned}
& {\left[\varepsilon_{1}(t)-\varepsilon_{2}(t)\right] \cos 2 \gamma(t)=C_{\varepsilon c}^{-1}(0) \cdot n(0) \cos 2 \alpha(0)} \\
& \quad+\int_{0}^{t} C_{\varepsilon c}^{-1}(t-\tau) \frac{d n(\tau) \cos 2 \alpha(\tau)}{d \tau} d \tau
\end{aligned}
$$

$$
\begin{aligned}
& {\left[\varepsilon_{1}(t)-\varepsilon_{2}(t)\right] \sin 2 \gamma(t)=C_{\varepsilon c}^{-1}(0) \cdot n(0) \sin 2 \alpha(0)} \\
& \quad+\int_{0}^{t} C_{\varepsilon c}^{-1}(t-\tau) \frac{d n(\tau) \sin 2 \alpha(\tau)}{d \tau} d \tau
\end{aligned}
$$

here, $C_{\sigma r}^{-1}(t)$ is the inverse relaxation stress-birefringence coefficient and $C_{\varepsilon c}^{-1}(t)$ is the inverse creep strainbirefringence coefficient which were measured in advance, as shown in the previous paper ${ }^{(2)}$.

\subsection{Diagonal summation theorem}

The superposition of two different photoelastic images obtained through a plane polariscope with polarization angles differing by $\pi / 4$ separates isochromatics from isoclinics, and is called the diagonal summation theorem ${ }^{(6)}$. The brightness distribution of the image $I(\theta)$ through a plane polariscope is given by

$$
I(\theta)=a^{2} \sin ^{2} 2(\varphi-\theta) \sin ^{2}(N \pi)
$$

where $a$ is the amplitude of incident polarized light, $N$ is the fringe order of isochromatics, $\varphi$ is the angle of the principal stress direction and $\theta$ is the angle of the polarization axis of incident light. For the two angles $\theta_{1}$ and $\theta_{2}$ where $\theta_{1}-\theta_{2}=\pi / 4$, the following calculations are easily performed.

$$
\begin{aligned}
& I\left(\theta_{1}\right)+I\left(\theta_{2}\right)=a^{2}\left\{\sin ^{2} 2\left(\varphi-\theta_{1}\right)\right. \\
& \left.\quad+\sin ^{2} 2\left(\varphi-\theta_{2}\right)\right\} \sin ^{2}(N \pi) \\
& \quad=a^{2}\left\{\sin ^{2} 2\left(\varphi-\theta_{1}\right)\right. \\
& \left.\quad+\sin ^{2} 2\left(\varphi-\theta_{1}+\theta_{1}-\theta_{2}\right)\right\} \sin ^{2}(N \pi) \\
& \quad=a^{2}\left\{\sin ^{2} 2\left(\varphi-\theta_{1}\right)+\cos ^{2} 2\left(\varphi-\theta_{1}\right)\right\} \sin ^{2}(N \pi) \\
& \quad=a^{2} \sin ^{2}(N \pi)
\end{aligned}
$$

Thus, we have,

$$
\varphi=\theta_{1}+\frac{1}{4} \cos ^{-1}\left\{1-\frac{2 I\left(\theta_{1}\right)}{I\left(\theta_{1}\right)+I\left(\theta_{2}\right)}\right\}
$$

at every point where $\sin (N \pi) \neq 0$.

Unlike the case of photoelasticity, it should be noted that the principal directions of stress, strain and birefringence in photoviscoelasticity are not coincident with each other particularly under the general condition of nonproportional load. For photoviscoelastic image, the fringe order $N$ and principal stress direction $\varphi$ in Eq. (3) for the photoelastic case must be adapted to the time-dependent fringe order $n(t)$ and the principal direction of birefringence $\alpha(t)$, respectively. Thus, in order to analyze viscoelastic stress and strain through a plane polariscope on the basis of Eqs. (1) and (2), the variations of a set of two photoviscoelastic images at different polarization angles $\theta_{1}$ and $\theta_{2}$ should be recorded. Utilizing Eqs. (4) and ( 5 ) with Eqs. (1) and (2) in the case of the photoviscoelastic image, not only the time-dependent difference of principal stresses $\sigma_{1}(t)-\sigma_{2}(t)$ and principal strain difference $\varepsilon_{1}(t)-\varepsilon_{2}(t)$, but also their timedependent principal directions, $\beta(t)$ and $\gamma(t)$, are easily calculated.

\section{3 Material properties}

The material used is made of an epoxy resin (Epikote 871) mixed with a hardener (triethylene- 
tetramine) in the weight ratio of 10 to 1 , then cured at a temperature of $353 \mathrm{~K}$. The glass transition temperature of the material is measured as $258 \mathrm{~K}$. Figure 1 shows the photoviscoelastic characteristics of the material adopted, i.e., the inverse relaxation stress ${ }^{-}$ birefringence coefficient $C_{\sigma r}^{-1}(t)$ and the inverse strainbirefringence coefficient $C_{c c}^{-1}(t)$. These coefficients were measured and determined through precise uniaxial tension tests under a constant strain rate at several temperatures; then they were approximated numerically utilizing the function of prony series for later use in the calculation of convolution integrals involved in Eqs. (1) and (2).

\section{4 Experimental procedure}

The shape of the specimen employed was a strip,

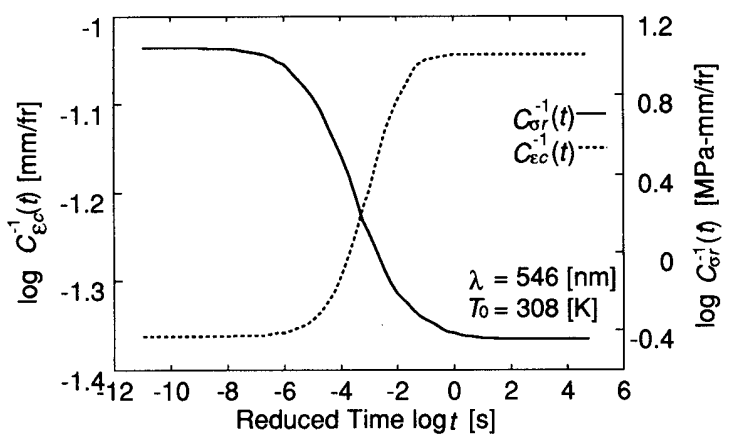

Fig. 1 Master curves of $C_{\sigma r}^{-1}(t)$ and $C_{\varepsilon c}^{-1}(t)$ of the material

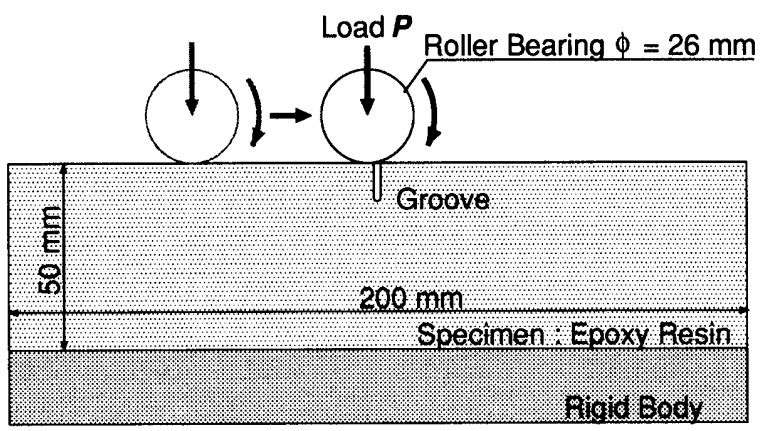

(a) Specimen geometry

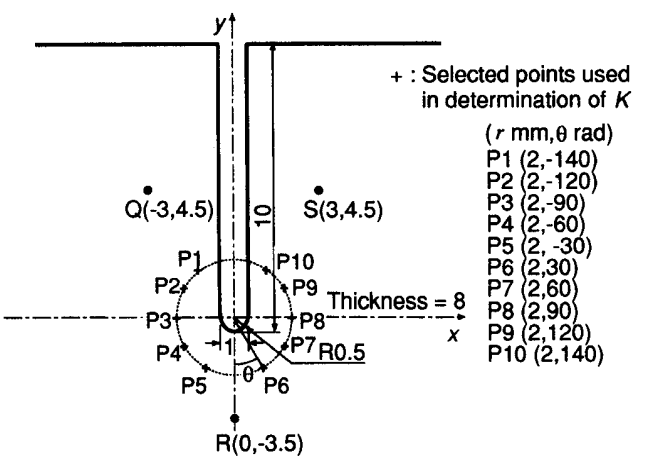

(b) Surface groove and selected points used in analysis

Fig. 2 Schematic diagrams of experimental setup
$200 \mathrm{~mm}$ in width, $50 \mathrm{~mm}$ in height and $8 \mathrm{~mm}$ in thickness, with a groove at the center of the upper edge, as shown in Fig. 2. The groove was $1 \mathrm{~mm}$ in width and $10 \mathrm{~mm}$ in depth with a $0.5 \mathrm{~mm}$ radius of the tip. Experiments were conducted at the temperature $T=273 \mathrm{~K}$ where the material shows marked viscoelastic behavior. The load $P=37.1 \mathrm{~N}$ was applied by a cylindrical steel roller moving over the groove at the constant speed $v=0.13 \mathrm{~mm} / \mathrm{s}$. The constant temperature was carefully maintained during the experiment within $\pm 0.5 \mathrm{~K}$.

Photoviscoelastic images were recorded using a CCD camera of $512 \times 480$ pixels as digital images of 256 grey levels of brightness through a plane polariscope with monochromatic light of $\lambda=546 \mathrm{~nm}$ emitted by a mercury lamp through a green filter.

Three points, Q, R and S, as shown in Fig. 2, were selected to evaluate the time dependence of principal stress and strain and their directions in the vicinity of the groove tip. Ten points on the small circle of radius $2 \mathrm{~mm}$, as shown, were also selected to evaluate stress intensity factors.

\section{Finite-Element Analysis}

The results of photoviscoelastic analysis were compared with those of FEM viscoelastic analysis. The element used was a 4-node plane stress quadrilateral, and the mesh distribution and boundary conditions are shown in Fig. 3. The numbers of nodes and elements of the model were 1432 and 1430 , respectively. Displacement at the bottom of the model was fixed in the $x$ and the $y$ direction. A rigid roller was slowly pressed onto the upper edge of the plate specimen at a location $39 \mathrm{~mm}$ left of the groove and loaded up to $0.5 \mathrm{~mm}$ displacement in depth during a period of $0.1 \mathrm{~s}$ $\times 10$ steps. Then the roller was moved slowly toward

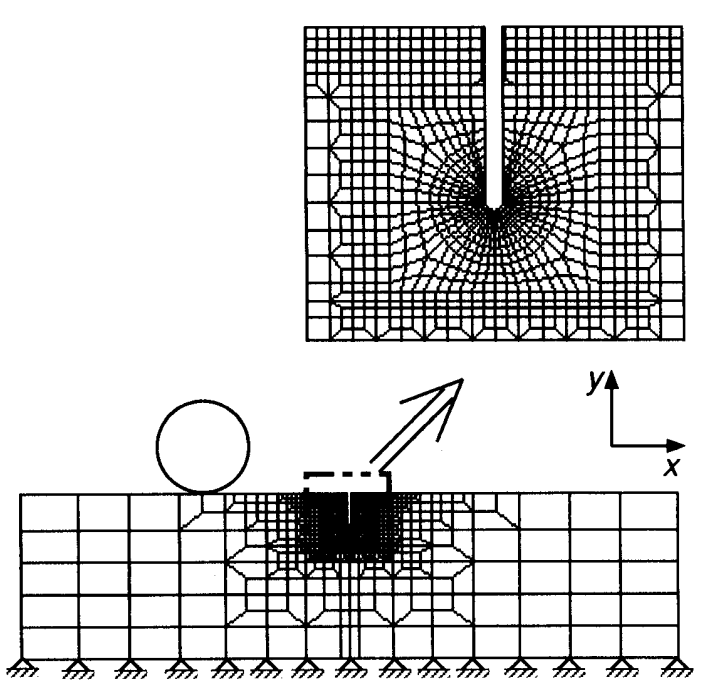

Fig. 3 Finite-element modeling and boundary conditions 
the groove at a velocity of $0.13 \mathrm{~mm} / \mathrm{s}$ from the initial contact point to the right-hand side of the specimen during a $0.1 \mathrm{~s} \times 600 \mathrm{step}$ period. The friction between the roller and the specimen was neglected.

The mechanical property adopted for analysis, i.e., the relaxation modulus $E_{r}(t)$, was approximated with an 11-term prony series obtained from the master curve which was measured previously ${ }^{(2)}$. Numerical results of the principal stress difference and principal strain difference were obtained using the viscoelastic constitutive relation in the relaxation form which was employed in the MARC finite-element program.

\section{Determination of Stress Intensity Factors from Principal Stress Difference}

Sanford and Dally ${ }^{(7)}$ proposed a method for calculating mixed-mode stress intensity factors $K_{\mathrm{I}}$ and $K_{\mathrm{II}}$ from isochromatic fringes near the crack tip using the least-squares solution in the framework of photoelastic analysis. Referring to their approach to a hyperbolic notch ${ }^{(8)}$, the relationship between principal stress difference and stress intensity factors is expressed as

$$
\left(\sigma_{1}-\sigma_{2}\right)^{2}=\frac{1}{2 \pi r}\left[-\frac{K_{1} \rho}{r} \cos \frac{3 \theta}{2}\right.
$$

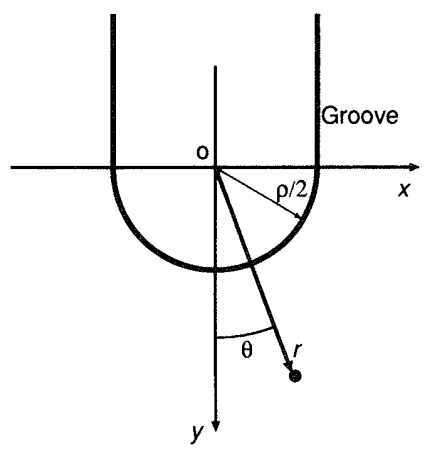

Fig. 4 Polar coordinate system at the groove tip

$$
\begin{aligned}
& -K_{\mathrm{I}} \cos \frac{\theta}{2}\left(1-\sin \frac{\theta}{2} \sin \frac{3 \theta}{2}\right) \\
& \left.+\frac{K_{\mathrm{II}} \rho}{r} \sin \frac{3 \theta}{2}-2 K_{\mathrm{II}} \sin \frac{\theta}{2}\left(1+\cos \frac{\theta}{2} \cos \frac{3 \theta}{2}\right)\right]^{2} \\
& +\frac{4}{2 \pi r}\left[-\frac{K_{\mathrm{I}} \rho}{2 r} \sin \frac{3 \theta}{2}+K_{\mathrm{I}} \cos \frac{\theta}{2}\left(\sin \frac{\theta}{2} \sin \frac{3 \theta}{2}\right)\right. \\
& \left.-\frac{K_{\mathrm{II}} \rho}{2 r} \cos \frac{3 \theta}{2}+K_{\mathrm{II}} \cos \frac{\theta}{2}\left(1-\sin \frac{\theta}{2} \sin \frac{3 \theta}{2}\right)\right]^{2}
\end{aligned}
$$

where $K_{\mathrm{I}}$ and $K_{\mathrm{II}}$ are mode I and mode II stress intensity factors respectively, $r$ and $\theta$ are polar coordinates with the origin defined at a distance $\rho / 2$ away from the groove tip, as shown in Fig. 4. In the case of viscoelasticity, it is expected that time-dependent stress intensity factors are obtained utilizing Eq. (6) with Eqs. (1) and (2).

\section{Results and Discussions}

\section{1 Photoviscoelastic fringe pattern}

Examples of the variations of photoviscoelastic fringe patterns around the surface groove with time at $T=273 \mathrm{~K}$ are shown in Fig. 5 ( a ). Figure 5 ( $\mathrm{b}$ ) shows image data of the principal birefringence direction constructed by the theorem mentioned in section 2 . For the array in Fig. 5(a), as the roller travels toward the mouth of the groove, the fringe order at the groove tip increases markedly up to $t=186 \mathrm{~s}$, and stress concentration at the groove tip also increases. In addition, the interaction of fringes generated at the point of contact and the groove tip becomes stronger. On the other hand, when the roller is right above the groove, the fringe order decreases suddenly at the groove tip. After the roller toward the right-hand side of the groove, the fringe order increases rapidly once more then decreases gradually.

\section{2 Differences of principal stresses and strains}

The variations of fringe order $n(t)$ and of principal birefringence direction $\alpha(t)$ at the points $\mathrm{Q}, \mathrm{R}$ and $\mathrm{S}$ shown in Fig. 2 were measured using the procedure
$85 \mathrm{~s}$
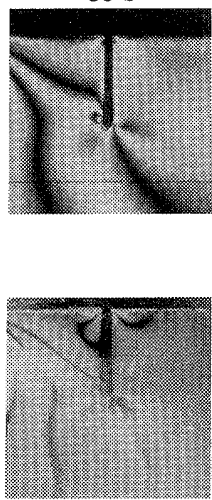

$186 \mathrm{~s}$

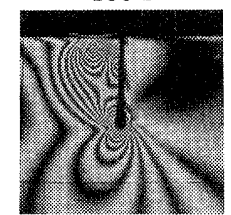

$210 \mathrm{~s}$

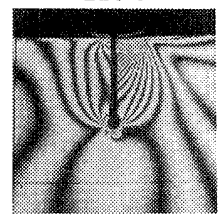

(a) Isochromatics

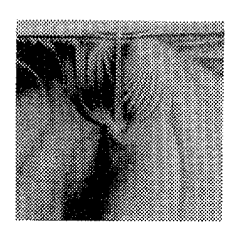

$233 \mathrm{~s}$
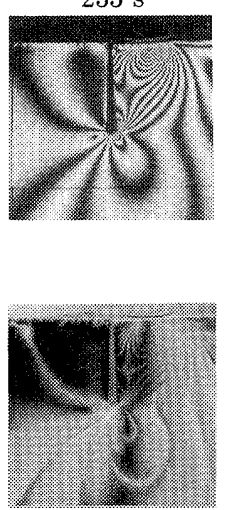
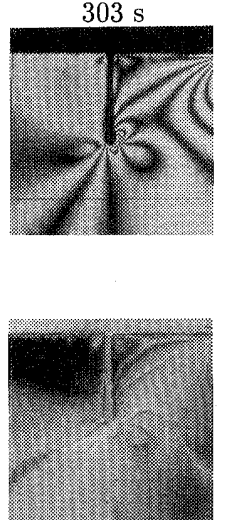

(b) Image data of principal direction of birefringence

Fig. 5 Variation of photoviscoelastic fringe pattern 
mentioned in section 2, as shown in Figs. 6 and 7, respectively. The variations of fringe order at the points $\mathrm{Q}$ and $\mathrm{S}$ do not show good symmetry with respect to the time $t=210 \mathrm{~s}$ at which the roller passes right above the groove. Particularly at the point $\mathrm{S}$, the rate of stress increase seems unexpectedly rapid when the roller passes over the groove. At the point $\mathrm{R}$, just beneath the groove tip, the fringe order does not increase greatly, and two extrema of the fringe order are observed before and after the time at which the roller passes right above the groove.

With regard to the time variation of the principal birefringence direction, it is the lower the temperature becomes, the steeper the direction changes, particularly at the point $\mathrm{S}$ on the right-hand side of the specimen.

The time variations of the principal stress difference $\sigma_{1}(t)-\sigma_{2}(t)$ and of the direction $\beta(t)$ with time are shown in Figs. 8 and 9, and those of the principal strain difference $\varepsilon_{1}(t)-\varepsilon_{2}(t)$ and the direction $\gamma(t)$ in Figs. 10 and 11, respectively. It is noteworthy, particularly in Fig. 8 , the values of principal stress difference at the point $S$ is much higher than that at the point $\mathrm{Q}$, due to the rapid increase of the

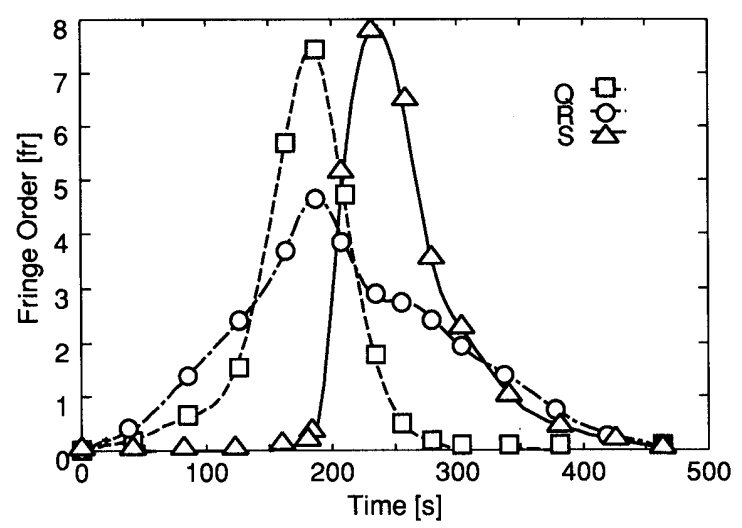

Fig. 6 Time variation of fringe order $n(t)$

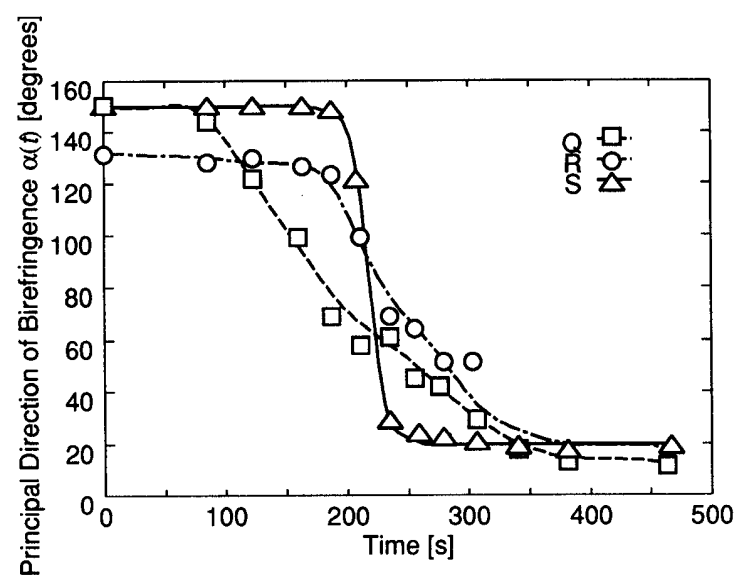

Fig. 7 Time variation of principal direction of birefringence $\alpha(t)$

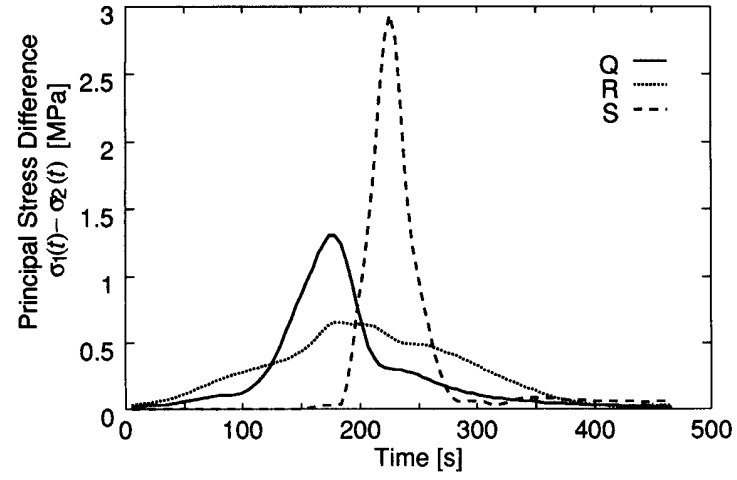

Fig. 8 Time variation of principal stress difference $\sigma_{1}(t)-\sigma_{2}(t)$

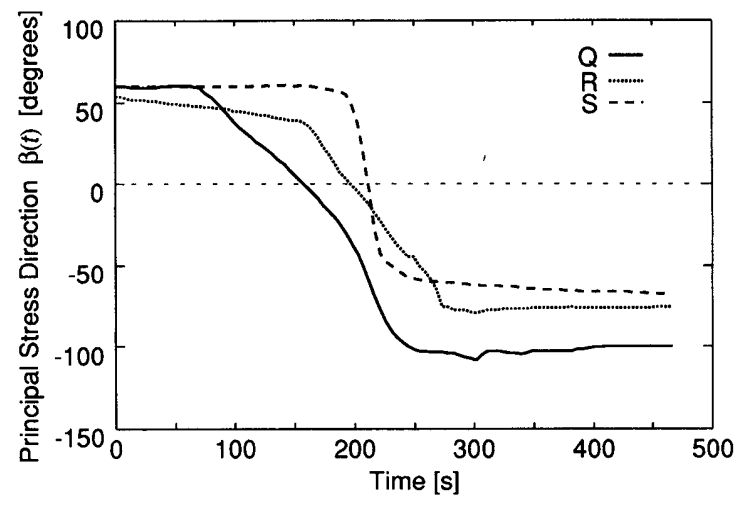

Fig. 9 Time variation of principal stress direction $\beta(t)$

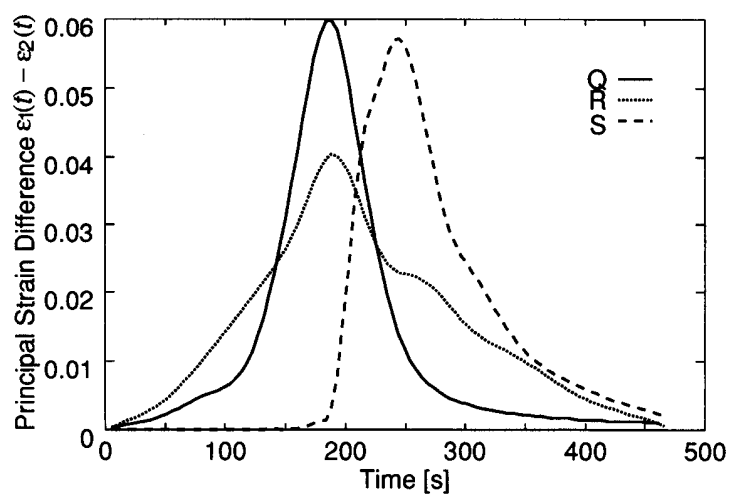

Fig. 10 Time variation of principal strain difference $\varepsilon_{1}(t)-\varepsilon_{2}(t)$

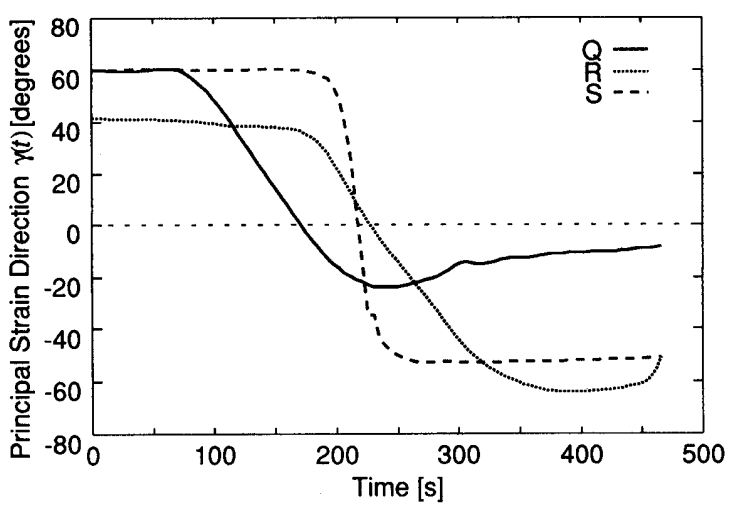

Fig. 11 Time variation of principal strain direction $\gamma(t)$ 


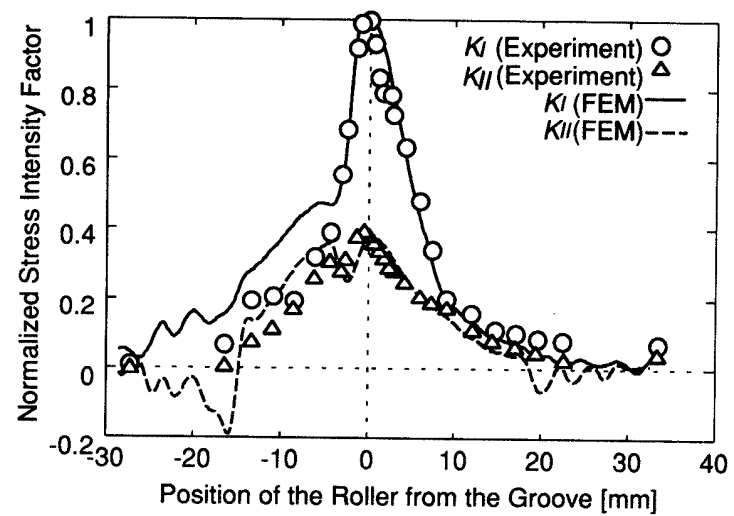

Fig. 12 Variation of stress intensity factors $K_{I}$ and $K_{I I}$

fringe order and rapid change in the principal birefringence direction which reflect the marked viscoelastic response of the material. At the point $\mathrm{R}$, the time variation of the stress difference is not symmetric about the time at which the roller passes over the groove. The principal strain difference shows a very similar variation as that of the fringe order, regardless of location and temperature.

Taking all the phenomena observed in this experiment into account, it is pointed out that the mode I and mode II stress states arise simultaneously at the groove tip. Then, the sign of shearing stress changes rapidly as the roller travels immediately above the groove. This might be closely related to the two extrema appearing before and after the time at which the roller travels over the groove. Although, at first glance, a fairly low fringe order is seen at the point $\mathrm{R}$, just beneath the groove tip, it is unreasonable to conclude that stress concentration at that point is not high, because the fringe order corresponds only to the principal stress difference, not to the principal stress itself.

\section{3 Stress intensity factors}

In Fig. 12, the variation of stress intensity factors $K_{\mathrm{I}}$ and $K_{\mathrm{II}}$ at the groove tip obtained by the method described in section 4 is shown, together with the results calculated by FEM. The ordinate gives the stress intensity factor and the abscissa represents the position of the cylindrical roller from the center of the groove. The results obtained in the experiment and by FEM show fairly good agreement.

Although the principal stress difference at the groove tip is small when the roller is right above the groove, stress intensity factors $K_{I}$ and $K_{I I}$ at that time increase. Although the photoviscoelastic method proposed as Eq. ( 6 ) cannot be used to determine the sign of the stress intensity factor, it is pointed out that the value of $K_{I}$ must be negative in this experiment since the groove is closed and compressive stress arises at the groove tip. Thus, the mode II stress state might be more dominant than the mode I stress state in the crack growth process at the groove tip.

\section{Concluding Remarks}

Using a new computer-aided photoviscoelastic technique, an experimental approach to the rolling contact problem between a viscoelastic strip having a deep surface groove and a hard cylindrical roller was discussed in the framework of photoviscoelasticity. Not only the time-dependent principal stress difference and principal strain difference but their directions under the nonproportional loading condition were evaluated successfully and accurately. A marked viscoelastic behavior of the material was observed in terms of the time variation of the principal stress difference at low temperature on the righthand side of the specimen. In addition, time-dependent stress intensity factors were evaluated using the least-squares method following the technique proposed by Sanford and Dally ${ }^{(7)}$.

\section{Acknowledgments}

The authors appreciate the financial support of the Science Research Promotion Fund of the Japan Private School Promotion Foundation. Some experimental work was also supported by a 1995 Grant-inAid for Scientific Research from the Ministry of Education, Science and Culture and by the Center for Science and Engineering Research Institute, Aoyama Gakuin University. Our thanks are extended to Mr. $\mathrm{S}$. Yonemoto for his help in the fracture mechanics analysis.

\section{References}

(1) Christensen, R.M., Theory of Viscoelasticity, (1982), Academic Press Inc.

(2) Gotoh, J., Horie, H., Misawa, A. and Takashi, M., Photoviscoelastic Method for Time Dependent Stress and Strain Analysis under Non-proportional Loading, Proc. 1995 SEM Spring Conf. on Exp. Mech.,(1995), p. 234.

(3) Ayame, D., Gotoh, J., Misawa, A. and Takashi, M., Photoviscoelastic Stress Analysis in a Strip Plate under Rolling Contact, Proc. 1st Int. Conf. on Mech. of Time Dependent Materials, (1995), p. 218.

(4) Ayame, D., Gotoh, J. and Takashi, M., Stress Analysis around Contact Region in Viscoelastic Plate with Rigid Cylinder, Proc. 72nd JSME Spring Annual Meeting(VI), (1995), p. 102.

( 5 ) Kunio, T., Miyano, Y. and Sugimori, S., Fundamentals of Photoviscoelastic Technique for Analy. sis of Time and Temperature Dependent Stress and Strain, Applied Stress Analysis, T.H. Hyde and E. Ollerton, Eds., (1990), p. 588, Elsevier Applied Science. 
(6) Takashi, M., Mawatari, S., Toyoda, Y. and Kunio, T., A New Computer Aided System for Photoelastic Stress Analysis with Structure-driven Type Image Processing, Applied Stress Analysis, T.H. Hyde and E. Ollerton, Eds., (1990), p. 516, Elsevier Applied Science.

(7) Sanford, R.J. and Dally, J.W., A General Method for Determining Mixed-mode Stress Intensity Factors from Isochromatic Fringe Patterns, Eng. Fract. Mech., Vol. 11,(1979), p. 621.

(8) Creager, M. and Paris, P.C., Elastic Field Equations for Blunt Cracks with Reference to Stress Corrosion Cracking, Int. J. Fract. Mech., Vol. 3, (1967), p. 247. 